\title{
Stefan-Boltzmann'S Law under Relativistic Conditions; Generalized Case
}

\author{
E.V.Veitsman \\ Veitsman's Science Project, 28 Apartment, 5 Klimashkin Str., Moscow, 123557, Russia \\ *Corresponding Author: evveitsman@gmail.com
}

Copyright $@ 2013$ Horizon Research Publishing All rights reserved.

\begin{abstract}
An expression was obtained for the energy density of the moving black-body radiation, i.e., the Stefan-Boltzmann law valid in the interval of object velocities from zero to the velocity of light in vacuo. The object temperature is shown to comprise two parts. The first one is a scalar invariant under the Lorentz transformations. The second one is a vector depending on the velocity of system motion. The scalar component of the temperature is a contraction of two tensor components of rank 3. Under normal conditions this mathematical object is a scalar. Taking account of a tensor character of the temperature a new formulation is given for the second thermodynamics law. The results obtained are of the great practical importance, in particular, while designing devices to measure the radiation temperature of moving cosmic objects, e.g., quasars.
\end{abstract}

Keywords Black Body, Radiation, Stefan-Boltzmann Law, Temperature, Special Relativity

\section{Introduction}

The problem of the moving black-body radiation arose in 1907 - almost immediately after the creation of Special relativity (SR). It is in this year that Kurd von Mosengeil's big article was published in der Annalen der Physik [1]. This work supervised by Max Planck underlies his relativistic thermodynamics [2]. The great scientist considered the theory of the black-body radiation to be well-studied and the most suitable for formulating foundations of thermodynamics correct over the entire whole interval of object velocities v, i.e., ranging from zero to the velocity of light in vacuo.

In article [1] a system is studied comprising a radiator of electromagnetic waves, receiver and reflector (mirror). The radiators are receivers at the same time. The three elements are moving uniformly and rectilinearly in space with a relativistic velocity forming an acute angle with one another. As a result, the temperature transformation law was obtained under relativistic conditions:

$$
T=T_{0} \sqrt{1-\beta^{2}}
$$

where $T_{0}$ is the temperature if $\mathrm{v}<<\mathrm{c}$ (here and below index " 0 " means that the given quantity concerns normal conditions); $\beta=v / c$.

For more than 50 years formula (1) had not been called in question until X.Ott's article was published [3], in which the relativistic temperature was shown to transform following another law:

$$
T=T_{0} / \sqrt{1-\beta^{2}} .
$$

The expression (2) was obtained by X.Ott for a variety of physical processes including electromagnetic radiation. However unlike Mosengeil, X.Ott elected another approach for studying the process of electromagnetic wave radiation under relativistic conditions. He examined wave emission of individual atoms, whereas Mosengeil studied black-body radiation, as we have noticed above. In particular, in [1] Stefan-Boltzmann's law was obtained:

$$
\varepsilon_{0}=\frac{E_{0}}{V_{0}}=a T_{0}^{4},
$$

based on the famous Planck formula derived first semiempirically:

$$
\rho(\omega, T) d \omega=\frac{8 \pi h \omega^{3} d \omega}{c^{3}\left(e^{\frac{h \omega}{k T}}-1\right)},
$$

where $E_{0}$ is the radiation energy of the black-body; $V_{0}$ is the volume; $a$ is Stephan-Boltzmann's constant $\left(\mathrm{J} / \mathrm{cc} \cdot \operatorname{grad}^{4}\right)$; $\rho(\omega, T)$ is the radiative energy density $(\mathrm{J} / \mathrm{cc}) ; k$ is Boltzmann's constant; $\omega$ is the frequency of oscillator radiation.

As known, Stefan-Boltzmann's constant equals:

$$
a=\frac{k^{4} \pi^{2}}{15 \hbar^{3} c^{3}} \text {. }
$$


X.Ott's article has induced a long-term polemic on the temperature transformation under relativistic conditions. Some researchers adhered to Planck-Einstein's viewpoint; the others adhered to X.Ott's. Some scientists considered the temperature to be a relativistic invariant [4]. There appear absolutely exotic opinions. For example, the authors of Ref. [5] arrived at a conclusion of the temperature under relativistic conditions being changed both according to Planck, and to Ott, and to Callen and Horwitz as the able situation requires. Moreover, P.Landsberg and G.Matsas have decided to put end to the long-time dispute $[6,7]$. In particular, they write (I cite): “...the proper temperature T alone is left as the only temperature of universal significance. This seems to complete a story started 90 years ago [8] (more than 100 years today - E.V.) of how usual temperature transforms, and to conclude a controversy [3] of 33 years' standing". ( 50 years' today).

What is authors' opinion [6, 7] based on? Their basis is as follows.

First of all, the authors used an Unruh-De Witt detector, i.e., a two-level monopole, with a unit interval of the radiation energy $\hbar \omega^{\prime}$. Then the authors $[6,7]$ suppose that black-body radiation with the proper temperature $T$ is at rest in some inertial reference frame $S$. The excitation rate of the detector moving with a constant velocity $v$ is found from quantum field theory. It is proportional to the particle number density $n^{\prime}\left(\omega^{\prime}, T, v\right) d \omega^{\prime}$. As a result, the following formula was obtained:

$$
\begin{aligned}
& n^{\prime}\left(\omega^{\prime}, T, v\right) d \omega^{\prime}=\frac{\omega^{\prime} k T \sqrt{1-v^{2} / c^{2}}}{4 \pi^{2} c^{2} v \hbar} \\
& \ln \left(\frac{1-e^{-\left(\hbar \omega^{\prime} \sqrt{1-v / c}\right) / k T \sqrt{1-v / c}}}{1-e^{-\left(\hbar \omega^{\prime} \sqrt{1-v / c}\right) / \sqrt{1+v / c}}}\right) d \omega^{\prime}
\end{aligned}
$$

which, as the authors of $[6,7]$ noted, could not be reduced at $v=0$ to the well-known formula

$$
n^{\prime}\left(\omega^{\prime}, T^{\prime}, v\right) d \omega^{\prime}=\frac{\omega^{2} / c^{3}}{2 \pi^{2}\left(e^{\hbar \omega^{\prime} / k T^{\prime}}-1\right)} d \omega^{\prime} .
$$

In opinion of P.Landsberg and G.Matsas, formula (6) is absolutely correct, thus it is unnecessary to speak about a unified law of temperature transformation under relativistic conditions. However it is not completely the case. Both the results obtained by Mosengeil (and soon used by Planck), and the mathematical monster (6) are incorrect. It is necessary to admit that the main reason of such a dramatic situation with a relativistic temperature is a giant scientific authority of Max Planck first and Albert Einstein. Naturally, after publishing X.Ott's article this work was carefully checked. Errors had not been found. But nobody dared check the works $[1,2,8]$. These articles were carried out just after the creation of Special Relativity (SR) when nobody had known on the Bose-Einstein distribution. As we have noticed above, Planck's well-known formula, concerning black-body radiation, was obtained by a semiempirical way without involving this distribution. After the discovery of this distribution, in the twenties of last century, Planck's formula was already obtained with its help. However if the radiator of electromagnetic waves is moving with a relativistic velocity, the form of Bose-Einstein distribution changes drastically - it becomes at least a function of two variables, which immediately follows from SR electrodynamics.

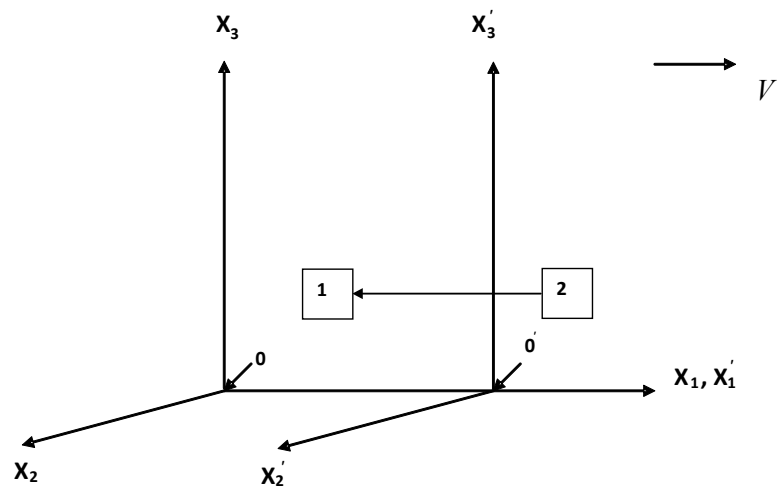

Figure 1. $\mathrm{X}_{1}, \mathrm{X}_{2}, \mathrm{X}_{3}$ and $\mathrm{X}_{1}^{\prime}, \mathrm{X}_{2}^{\prime}, \mathrm{X}_{3}^{\prime}$ are the laboratory reference frame and that moving uniformly and rectilinearly with the velocity $\mathrm{v} .1$ is the observer at rest; 2 is the radiating black body

Indeed, examine the simplest case represented in the Fig.1. As seen, there are two reference frames. One of them (with primes) is moving uniformly and rectilinearly with the velocity $\mathbf{v}$. A photon radiator is at rest in the moving reference frame. An observer is at rest in the laboratory reference frame. The observer is detecting photons. If the angle $\theta$ between $\mathbf{v}$ and the observer is zero, then the radiation frequency of the oscillator $\omega$ will be for this case equal to

$$
\omega=\omega_{0} \frac{\sqrt{1-\beta^{2}}}{1+\beta} .
$$

If the angle were $\pi / 2$, so the formula for the frequency transformation would have another form, namely:

$$
\omega=\omega_{0} \sqrt{1-\beta^{2}} .
$$

for the observer in the laboratory reference frame.

Thus without taking into consideration (8) and (9), we cannot evidently use the well-known Bose-Einstein distribution for obtaining the Stefan-Boltzmann law when the object under study is moving with relativistic velocities.

The aforesaid allows us to formulate a main goal of our work - obtaining a radiation law for the black-body moving with a relativistic velocity when the angle $\theta$ between the moving velocity $\mathbf{v}$ and the observer is zero (see Fig.1) and when it can have any values (from zero up to $\pi / 2$; see Fig.2). A solution of the problem will be performed by the methods given in [9]. It can say: having solved the second 
subproblem, we, in fact, solved the first one, since the solution also contained the case when the angle $\theta$ was equal to zero. However, as we shall see below, the solution of the problem in the first case will differ from the solution in the second case when the angle is equal to zero as well. Why? Because the first subproblem is solved in the cylindrical space; the second one is done in Cartesian space.

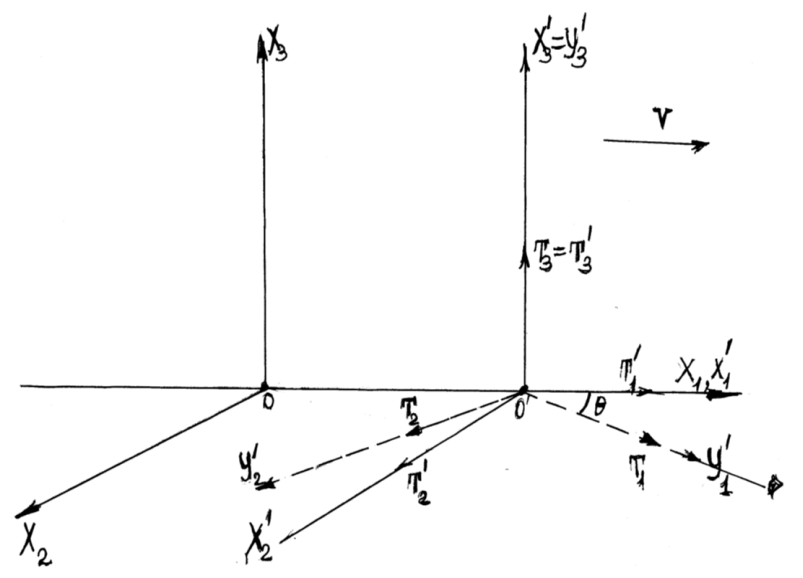

Figure 2. $X_{1}, X_{2}, X_{3}$ is the laboratory reference frame; $X_{1}{ }^{\prime}, X_{2}{ }^{\prime}, X_{3}{ }^{\prime}$ and $Y_{1}{ }^{\prime}$, $Y_{2}^{\prime}, Y_{3}^{\prime}$ are the reference frames moving with a velocity v. There is a black body radiating electromagnetic waves at the point $0^{\prime} . T_{1}, T_{2}, T_{3}$ and $T_{1}^{\prime}, T_{2}^{\prime}$, $\mathrm{T}_{3}$, are the projections of the black body temperature on the coordinate axes $\mathrm{X}_{1}^{\prime}, \mathrm{X}_{2}^{\prime}, \mathrm{X}_{3}^{\prime}$ and $\mathrm{Y}_{1}^{\prime}, \mathrm{Y}_{2}^{\prime}, \mathrm{Y}_{3}^{\prime}$

Here we must be added the following. Attempts have been made to obtain the law connecting the radiation intensity with the temperature when relativistic effects are involved $[10,11]$. For example, in [11] an ultrarelativistic plasma is examined containing electrons and positrons. Their annihilation generates electromagnetic radiation. Its intensity is defined, in particular, with the help of a one-dimensional Bose-Einstein distribution. It is proportional to the plasma temperature to the fourth power, with the velocity of the object as a whole being equal to zero. It is plasma particles that are in motion.

\section{Methods and Results}

\subsection{Definition of the Number of Field Oscillators with a Given Frequency when the Angle $\Theta$ Is Zero (Fig.1)}

Assume that we have an opaque object with an inner cylindrical cavity. Its surface is a black body heated up to some temperature $T$. There is a thermodynamical equilibrium in the cavity between its inner surface and electromagnetic radiation. There is a very small hole in the object cover, through which electromagnetic waves radiate out of the cavity (see Fig.1). The object is moving uniformly and rectilinear with the velocity $\mathbf{v}$ together with the reference frame. The radiation from the cavity is detected with a device being at rest in a laboratory reference frame. First of all, we will show that the Stefan-Boltzmann law (3) is incorrect over the whole range of object motion velocities, i.e., from zero up to $v \rightarrow c$. Indeed, according to X. Ott [3], the radiation energy in the cavity is equal to:

$$
E=\frac{\sum_{1}^{n} h \omega_{0}^{(n)}}{\sqrt{1-\beta^{2}}}, \quad n=1,2, \ldots . l,
$$

then the electromagnetic energy density

$$
\varepsilon=\frac{E_{0}}{V_{0}\left(1-\beta^{2}\right)}=\frac{\sum_{1}^{n} h \omega_{0}^{(n)}}{V_{0}\left(1-\beta^{2}\right)}, n=1,2, \ldots l,
$$

where $n$ is an oscillator serial number, $\omega^{(n)}$ is the frequency of its oscillations.

No matter how the temperature of the system transforms, i.e., according to Planck or to Ott or to Callen and Horwitz, we shall always arrive at the point of absurdity. Indeed, let the temperature transform, e.g., according to Planck, i.e., to (1). In this case the right side of (3) will have the following form $a T_{0}^{4}\left(1-\beta^{2}\right)^{2}$. Then, as seen from (11), the right side of (3) appears to tend to zero as $v \rightarrow c$, while the left side of this formula to increase infinitely. This indicates a close connection between the radiation law of a moving black body and the temperature transformation under relativistic conditions.

Now find the number of oscillators $g\left(\omega_{1}, \omega_{2}\right) d \omega_{1} d \omega_{2}$ with frequencies in intervals $\omega_{1}, \omega_{1}+d \omega_{1}$ and $\omega_{2}, \omega_{2}+d \omega_{2}$ and a given polarization in the cavity using the well-known procedure [9]. The following fact should be pointed out at once. The number of these oscillators is a function of two variables. The reason for that was explained above but here the following should be noted. If a spherical coordinate system is used for the case $v<<c$, then in our case it is convenient to use a cylindrical one taking account of formulae (8) and (9).

The classical approach to finding the quantity $g(\omega) d \omega$ is based on using the number space $n$ followed by transition to a spherical space of the wave vector $k=|\boldsymbol{k}|=n \frac{2 \pi}{L}$, where $L$ is the normalized cube edge, and finally to the spherical space of frequencies $\omega$. In the case studied we use a cylindrical space representable as two spaces - flat, circular and linear perpendicular to one another. Then to define the necessary quantity we shall use two coordinate systems: polar and one-dimensional Euclidean, i.e., a straight line. The amount of numbers within the spherical layer $d n$ of the spherical space is $4 \pi n^{2} d n$ [9] (the spherical coordinate system). The amount of numbers $n_{1}$ in the circular layer is equal to $2 \pi n_{1} d n_{1}$ (the polar coordinate system). As to $n_{2}$ in a linear interval of one-dimension space, it will be equal to $d n_{2}$. As a result, we have for the whole system: 


$$
g\left(\omega_{1}, \omega_{2}\right) d \omega_{1} d \omega_{2}=2 \pi n_{1} d n_{1} d n_{2} .
$$

Turning from a number space to a wave vector space and finally to a frequency one, we shall have:

$$
\begin{gathered}
g\left(\omega_{1}, \omega_{2}\right) d \omega_{1} d \omega_{2}= \\
2 \pi m_{1} d n_{1} d n_{2}=\frac{2 \pi k_{1} d k_{1} d k_{2}}{(2 \pi)^{3}} \Delta L_{1}^{2} \Delta L_{2}=\frac{\omega_{1} d \omega_{1} d \omega_{2}}{(2 \pi)^{2} c^{3}} \Delta L_{1}^{2} \Delta L_{2} \\
=\frac{\omega_{1} d \omega_{1} d \omega_{2}}{(2 \pi)^{2} c^{3}} \Delta V .
\end{gathered}
$$

In case of electromagnetic waves should be taken into account two polarizations, then we shall have:

$$
g\left(\omega_{1}, \omega_{2}\right) d \omega_{1} d \omega_{2}=\frac{\omega_{1} d \omega_{1} d \omega_{2}}{2 \pi^{2} c^{3}} \Delta V
$$

Here it is important to emphasize that formula (14) is correct for the observer at rest in a real space monitoring, from the referring frame, the object moving then uniformly and rectilinearly with the relativistic velocity v. Since the radiation is thermal the average volume of the oscillators with a given polarization will almost be independent of time. In this case, it is unnecessary to define oscillator numbers in Minkowski space.

\subsection{Relativistic Temperature as either a Vector or a Tensor}

Now we should make a new attempt to solve some problems connected with the relativistic temperature. First of all, we should clarify if this thermodynamic parameter is a scalar or appears to be a vector or a tensor. In this connection we should first recall the formulae for velocity addition in SR. As known, the components of the total velocity in the directions $\mathrm{X}_{2}$ or $\mathrm{X}_{3}$ will tend to zero for the observer in the laboratory reference frame as $v \rightarrow c$ (see Fig.1). In turn, the component parallel to axes the $X_{1}$ will not do. This suggests immediately that the temperature becomes a mathematical object different from a scalar. What is the object?

Until very recently the temperature in the above case is considered to be either a scalar or a quantity forming a vector with other quantities. For example, in [10] V. Hamity represents this thermodynamical parameter as

$$
\Theta^{\mu}=\frac{v^{\mu}}{\hat{T}}, \quad \mu=0,1,2,3,
$$

where $v^{\mu}$ is a unit 4-vector in Minkowski space, moreover

$$
v^{\mu}=\left[v^{0}, v^{\alpha}\right], \quad \alpha=1,2,3,
$$

i.e., $v^{\alpha} \equiv \mathrm{v}$ is a velocity vector in Euclidean space;

$$
v^{\mu} v_{\mu}=1 \text {. }
$$

Further, developing the idea of temperature vector representation, the author of [10] finally comes to the following expression:

$$
\beta_{\mu}=v_{\mu} / k T
$$

with $\beta_{\mu}=(\beta, 0,0,0)$, then

$$
\beta_{\mu}=\delta_{\mu}^{0} / k T, \quad \delta_{\mu}^{\nu}=\left(\begin{array}{llll}
1 & 0 & 0 & 0 \\
0 & 1 & 0 & 0 \\
0 & 0 & 1 & 0 \\
0 & 0 & 0 & 1
\end{array}\right) .
$$

Other authors, e.g., [12], also tried to represent the relativistic temperature exclusively as a vector. However, in our opinion, this approach to the problem is incorrect, since the photon gas in the cavity is a continuous medium. Then an expanded tensor approach is necessary to describe energy processes in it. In this case the second thermodynamics law can be represented in Minkowski space as

$$
\delta \sigma=\frac{\delta Q^{i j k} g_{j k}}{T^{i \alpha \beta} g_{\alpha \beta}} ; i, j, k=1,2,3,4 ; \alpha, \beta=1,2,3,4,
$$

where the heat $Q$ and the temperature $T$ are tensors of rank 3 , but $g_{j k}, g_{\alpha \beta}$ are covariant fundamental tensors.

Formula (20) needs a special explanation.

As known, M.Planck assumed that $\sigma \neq \sigma(\mathbf{v})$, i.e., the entropy of the system varies exclusively owing to thermodynamical processes in the object under study and is independent of its velocity relative to the observer in the laboratory reference frame [2]. As will be shown below, the law (20) agrees with the Planck statement. Further, the contraction of the heat and temperature tensors with the fundamental tensors transforms them to the vectors multiplied into scalar quantities. The latter are invariant parts of the above tensors that do not vary when passing from one reference frame to another. As to the vectors, their components are equal to unity when the moving system 4-velocity equals to zero, i.e.,

$$
\mathrm{n}=\left(\begin{array}{l}
1 \\
1 \\
1 \\
i
\end{array}\right)
$$

$$
n^{1}=\frac{n^{1}-\beta n^{4}}{\sqrt{1-\beta^{2}}}, n^{\prime 2}=n^{2}, n^{3}=n^{3}, n^{\prime 4}=\frac{-\beta n^{1}+n^{4}}{\sqrt{1-\beta^{2}}}
$$

where $i$ is imaginary unit; $\beta=v / c$,

Then the contraction in (20) of two vector quantities in indices $i$ gives a scalar quantity, which is invariant under the Lorentz transformations. As to heat and the temperature, their invariant parts vary exclusively owing to purely thermodynamic reasons. In turn, the vector components vary exclusively, when passing from one reference frame to another. In both cases either the heat or the temperature are 
inversely proportional to the quantity $\sqrt{1-\beta^{2}}$. Then the entropy will not change in the absence of heat input into the system. The latter is in a full accord with the results obtained in works [13,14, and 15] where the temperature was shown to transform under relativistic conditions in inverse proportion to the quantity $\sqrt{1-\beta^{2}}$. Then we can represent the temperature in Minkowski space as

$$
T^{i}=T^{i \alpha \beta} g_{\alpha \beta}=\mathrm{T} n^{i}=\mathrm{T} \mathbf{n},
$$

where $\mathrm{T}$ is the invariant part of the tensor magnitude of rank 3, i.e., $T^{i \alpha \beta}$. In the real space formulae (20) and (23) remain unchanged with the only difference that, first, we now use affine tensors, second, the dependences (21) and (22) take the form:

$$
\begin{gathered}
\mathrm{n}=\left(\begin{array}{l}
1 \\
1 \\
1
\end{array}\right), \\
n^{1}=\frac{n^{1}}{\sqrt{1-\beta^{2}}}, n^{2}=n^{2}, n^{\prime 3}=n^{3} .
\end{gathered}
$$

At $v=0$ the spatial components of $T^{i}$ coincide in Euclidean space with the same components in Minkowski space.

In space-time the components of squared sum of the vector quantity $\mathrm{T} \mathbf{n}$ read

$$
\mathrm{T}^{2} n_{x}^{2}+\mathrm{T}^{2} n_{y}^{2}+\mathrm{T}^{2} n_{z}^{2}=\mathrm{T}^{2} n_{\tau}^{2}=\mathrm{T}_{x}^{2}+\mathrm{T}_{y}^{2}+\mathrm{T}_{z}^{2}=\mathrm{T}_{\tau}^{2},(26)
$$

invariant in all inertial reference frames.

On the other hand the invariant of this sort gives in Euclidean space

$$
\begin{gathered}
\mathrm{T}^{2} n^{\prime 1} n_{1}^{\prime}+\mathrm{T}^{2} n^{\prime 2} n_{2}^{\prime}+\mathrm{T}^{2} n^{\prime 3} n_{3}^{\prime}= \\
=\mathrm{T}^{2} n^{1} n_{1}+\mathrm{T}^{2} n^{2} n_{2}+\mathrm{T}^{2} n^{3} n_{3}=\mathrm{T}_{x}^{2}+\mathrm{T}_{y}^{2}+\mathrm{T}_{z}^{2} \\
=\text { invar, }
\end{gathered}
$$

taking into consideration that $n_{1}^{\prime} n^{\prime 1}=1$ (affine tensors), i.e., the spatial part of the invariant connected with the temperature 4-tensor is completely identical to the invariant connected with the temperature 3-tensor. It is very important since it allows one to solve our problem directly in Euclidean space. As to the ultrarelativistic plasma considered in [11], the aforesaid will be valid in this case as well, which will be discussed below.

\subsection{The Radiation Intensity Dependence on the Temperature of the Black-Body Moving Uniformly and Rectilinearly. the Angle $\Theta$ Is Zero}

Based on the aforesaid as well as on the classical methods of solving the problem (i.e., for $v<<c$, see, e.g., $[9,16]$ ) we can now start its solving. For this purpose we shall write down an expression for the average total energy $\varepsilon$ of the linear oscillators as follows (cylindrical space, zero oscillations are neglected):

$$
\bar{\varepsilon}=\overline{\left(\varepsilon_{1}+\varepsilon_{2}\right)}=\frac{\hbar \sum_{n_{1}=0}^{\infty} \sum_{n_{2}=0}^{\infty}\left[\omega_{1} n_{1}+\omega_{2} n_{2}\right] e^{-\frac{\hbar \omega_{1} n_{1}}{k T_{1}}-\frac{\hbar \omega_{2} n_{2}}{k T_{2}}}}{\sum_{n_{1}=0}^{\infty} \sum_{n_{2}=0}^{\infty} e^{-\frac{\hbar \omega_{1} n_{1}}{k T_{1}}-\frac{\hbar \omega_{2} n_{2}}{k T_{2}}}}=
$$

$$
=\frac{\hbar \omega_{1} \sum_{n_{1}=0}^{\infty} n_{1} e^{-\frac{\hbar \omega_{1} n_{1}}{k T_{1}}} \sum_{n_{2}=0}^{\infty} e^{-\frac{\hbar \omega_{2} n_{2}}{k T_{2}}}+\hbar \omega_{2} \sum_{n_{1}=0}^{\infty} e^{-\frac{\hbar \omega_{1} n_{1}}{k T_{1}}} \sum_{n_{2}=0}^{\infty} n_{2} e^{-\frac{\hbar \omega_{2} n_{2}}{k T_{2}}}}{\sum_{n_{1}=0}^{\infty} e^{-\frac{\hbar \omega_{1} n_{1}}{k T_{1}}} \sum_{n_{2}=0}^{\infty} e^{-\frac{\hbar \omega_{2} n_{2}}{k T_{2}}}}=
$$

$$
\stackrel{n_{1}, n_{2}=1}{=} \frac{\hbar\left(\omega_{1}+\omega_{2}\right)}{e^{\frac{\hbar \omega_{1}}{\theta_{1}}} e^{\frac{\hbar \omega_{2}}{\theta_{2}}}-e^{\frac{\hbar \omega_{1}}{\theta_{1}}}-e^{\frac{\hbar \omega_{2}}{\theta_{2}}}+1}=\frac{\hbar\left(\omega_{1}+\omega_{2}\right)}{\left(e^{\frac{\hbar \omega_{1}}{\theta_{1}}}-1\right)\left(e^{\frac{\hbar \omega_{2}}{\theta_{2}}}-1\right)}
$$

where $\omega_{1}$ and $\omega_{2}$ are the frequencies of oscillators in the direction perpendicular and parallel to the velocity of the moving object; $n_{1}$ and $n_{2}$ are positive (quantum) integers for the oscillators in the first and second directions. In this case $n_{1}=n_{2}=1$, since photons are bosons, they can be in one quantum state; $T_{1}, T_{2}$ are the values of the temperature tensor components.

Then the average volume of the total energy $\varepsilon$ of the electromagnetic field per unit volume in the moving cavity proves to equal

$$
\begin{aligned}
& \varepsilon=\frac{E_{0}}{V_{0}\left(1-\beta^{2}\right)}=\frac{2 \hbar}{(2 \pi)^{2} c^{3}} \\
& \int_{0}^{\infty} \frac{\omega_{1}^{2} d \omega_{1}}{\left(e^{\frac{\hbar \omega_{1}}{\theta_{1}}}-1\right)} \int_{0}^{\infty} \frac{d \omega_{2}}{\left(e^{\frac{\hbar \omega_{2}}{\theta_{2}}}-1\right)}+\frac{2 \hbar}{(2 \pi)^{2} c^{3}} \int_{0}^{\infty} \frac{\omega_{1} d \omega_{1}}{\left(e^{\frac{\hbar \omega_{1}}{\theta_{1}}}-1\right)} \int_{0}^{\infty} \frac{\omega_{2} d \omega_{2}}{\left(e^{\frac{\hbar \omega_{2}}{\theta_{2}}}-1\right)}
\end{aligned}
$$

As a result, we have obtained, in fact, four improper integrals, three of them converge. The last two integrals in (29) differ only by variables. They are easily calculated using variable transformations as follows:

$$
\begin{gathered}
y_{1(2)}=\frac{\hbar \omega_{1(2)}}{\theta_{1(2)}}=\frac{\hbar \omega_{1(2)}}{k T_{1(2)}}, \\
I_{1}=\frac{2 \hbar}{(2 \pi)^{2} c^{3}} \int_{0}^{\infty} \frac{\omega_{1} d \omega_{1}}{\left(e^{\frac{\hbar \omega_{1}}{\theta_{1}}}-1\right)} \frac{\omega_{2} d \omega_{2}}{\left(e^{\frac{\hbar \omega_{2}}{\theta_{2}}}-1\right)}= \\
\frac{2 \theta_{1}^{2} \theta_{2}^{2}}{(2 \pi)^{2} \hbar^{3} c^{3}} \int_{0}^{\infty} \frac{y_{1} d y_{1}}{\left(e^{y_{1}}-1\right)} \int_{0}^{\infty} \frac{y_{2} d y_{2}}{\left(e^{y_{2}}-1\right)}=
\end{gathered}
$$


$=\frac{2 \theta_{1}^{2} \theta_{2}^{2}}{(2 \pi)^{2} \hbar^{3} c^{3}} \cdot \frac{\pi^{4}}{36}=\frac{k^{4} \pi^{2} T_{1}^{2} T_{2}^{2}}{72 \hbar^{3} c^{3}}=0.208 a T_{1}^{2} T_{2}^{2}$

where $a$ is the Stefan-Boltzmann constant, i.e.,

$$
\begin{gathered}
a=\frac{k^{4} \pi^{2}}{15 \hbar^{3} c^{3}} . \\
I_{2}=\frac{2 \hbar}{(2 \pi)^{2} c^{3}} \int_{0}^{\infty} \frac{\omega_{1}^{2} d \omega_{1}}{\left(e^{\frac{\hbar \omega_{1}}{\theta_{1}}}-1\right)} \frac{d \omega_{2}}{\left(e^{\frac{\hbar \omega_{2}}{\theta_{2}}}-1\right)}=\frac{2 \hbar}{(2 \pi)^{2}} I_{2}^{\prime} I_{2}^{\prime \prime} \\
I_{2}^{\prime}=\int_{0}^{\infty} \frac{\omega_{1}^{2} d \omega_{1}}{\left(e^{\frac{\hbar \omega_{1}}{\theta_{1}}}-1\right)}=\frac{\theta_{1}^{3}}{\hbar^{3}} \int_{0}^{\infty} \frac{y_{1}^{2} d y_{1}}{\left(e^{y_{1}}-1\right)}=\frac{\theta_{1}^{3}}{\hbar^{3}} \Gamma(z) \zeta(z)=\frac{\theta_{1}^{3}}{\hbar^{3}} \\
2 x 1.5498 \approx 3.1 \frac{\theta_{1}^{3}}{\hbar^{3}}
\end{gathered}
$$

where $\Gamma(z)$ is the gamma function, $z=2[17]$,

$$
\begin{gathered}
\zeta(z)=\sum_{k=1}^{\infty} \frac{1}{K^{z}}=1+\frac{1}{4}+\frac{1}{9}+\frac{1}{16}+\ldots \\
\approx 1.5498 ; \Gamma(z+1)=\Gamma(z) ; \Gamma(1)=\Gamma(2)=1 . \\
I_{2}^{\prime \prime}=\int_{0}^{\infty} \frac{d \omega_{2}}{\left(e^{\frac{\hbar \omega_{2}}{\theta_{2}}}-1\right)}= \\
\frac{\theta_{2}}{\hbar} \int_{0}^{\infty} \frac{d y_{2}}{\left(e^{y_{2}}-1\right)}=\frac{\theta_{2}}{\hbar}\left[-y+\ln \left(-1+e^{y}\right)\right]_{y=0}^{y \rightarrow \infty} .
\end{gathered}
$$

As seen, the integral (35) diverges and we take account of a high frequency radiation of the object. Having deleted -1 from the denominator, we obtain;

$$
I_{2}^{\prime \prime \prime}=\int_{0}^{\infty} e^{-\frac{\hbar \omega_{2}}{\theta_{2}}} d \omega_{2}=-\frac{\theta_{2}}{\hbar} \int_{0}^{\omega} e^{-y} d(-y)=\frac{\theta_{2}}{\hbar} .
$$

As a result, we have for $I_{2}$ :

$$
I_{2} \approx \frac{2 \hbar}{(2 \pi)^{2} c^{3}} I_{2}^{\prime} I_{2}^{\prime \prime \prime}=3.1 \frac{2 \theta_{1}^{3} \theta_{2}}{(2 \pi)^{2} \hbar^{3} c^{3}}=0.239 a T_{1}^{3} T_{2},
$$

and

$$
\begin{aligned}
& \varepsilon=\frac{E_{0}}{V_{0}\left(1-\beta^{2}\right)}=0.208 a T_{1}^{2} T_{2}^{2}+0.239 T_{1}^{3} T_{2}= \\
& =0.208 T_{(0) 1}^{2} \frac{T_{(0) 2}^{2}}{1-\beta^{2}}+0.239 T_{(0) 1}^{3} \frac{T_{(0) 2}}{\sqrt{1-\beta^{2}}}
\end{aligned}
$$

for the energy density of radiation under relativistic conditions.
Formula (38) will take the following form under normal conditions:

$$
\varepsilon=0.447 a T_{0}^{4} .
$$

The dissimilarity of formula (39) from the Stefan-Boltzmann law is quite natural, if one takes into account of the above assumption. To obtain a more exact expression for the black-body radiation under relativistic conditions, it is necessary to renormalize the dependence (39). The value of the numerical coefficient in (37) should be such that the new coefficient plus the coefficient from formula (31) would give unity.

Then we have finally:

$$
\begin{aligned}
& \varepsilon=\frac{E_{0}}{V_{0}\left(1-\beta^{2}\right)}=0.208 a T_{1}^{2} T_{2}^{2}+0.792 a T_{1}^{3} T_{2}= \\
& =0.208 a T_{(0) 1}^{2} \frac{T_{(0) 2}^{2}}{1-\beta^{2}}+0.792 a T_{(0) 1}^{3} \frac{T_{(0) 2}}{\sqrt{1-\beta^{2}}}
\end{aligned}
$$

This is the radiation law of the black-body being in a uniform and rectilinear motion when the angle $\theta$ between the velocity vector of the object $\mathbf{v}$ and the observer is equal to zero.

\subsection{Definition of the Number of Field Oscillators with a Given Frequency. The Angle $\Theta$ Has any Volume from Zero Up To $\Pi / 2$ (Fig. 2)}

Now find the number of oscillators $g\left(\omega_{1}, \omega_{2}, \omega_{3}\right)$ $d \omega_{1} d \omega_{2} d \omega_{3}$ with frequencies in intervals $\omega_{1}, \omega_{1}+d \omega_{1}$, $\omega_{2}, \omega_{2}+d \omega_{2}, \omega_{3}, \omega_{3}+d \omega_{3}$ and a given polarization in the cavity using the well-known procedure [9]. The following fact should be pointed out at once. The number of these oscillators is a function of three variables. The reason for that was explained above but here the following should be noted. If a spherical coordinate system is used for the case $v<<c$, then in our case it is convenient to use Cartesian system of coordinates taking account of the following formulae [9]

$$
\begin{aligned}
& \cos \theta=\frac{\cos \theta_{0}+\frac{v}{c}}{1+\frac{v}{c} \cos \theta_{0}} ; \\
& \sin \theta=\sin \theta_{0} \frac{\sqrt{1-\beta^{2}}}{1+\frac{v}{c} \cos \theta_{0}}
\end{aligned}
$$

In the case studied we use Cartesian coordinate system. Then we have for the system:

$$
g\left(\omega_{1}, \omega_{2}, \omega_{3}\right) d \omega_{1} d \omega_{2} d \omega_{3}=d n_{1} d n_{2} d n_{3} .
$$

Turning from a number space to a wave vector space and finally to a frequency one, we shall have:

$$
g\left(\omega_{1}, \omega_{2}, \omega_{3}\right) d \omega_{1} d \omega_{2} d \omega_{3}=
$$




$$
\begin{gathered}
d n_{1} d n_{2} d n_{3}=\frac{d k_{1} d k_{2} d k_{3}}{(2 \pi)^{3}} \Delta L_{1} \Delta L_{2} \Delta L_{3}= \\
=\frac{d \omega_{1} d \omega_{2} d \omega_{3}}{(2 \pi)^{2} c^{3}} \Delta L_{1} \Delta L_{2} \Delta L_{3} \\
=\frac{d \omega_{1} d \omega_{2} d \omega_{2}}{(2 \pi)^{2} c^{3}} \Delta V .
\end{gathered}
$$

In case of electromagnetic waves should be taken into account two polarizations, then we shall have:

$$
\begin{gathered}
g\left(\omega_{1}, \omega_{2}, \omega_{3}\right) d \omega_{1} d \omega_{2} d \omega_{3}= \\
\frac{d \omega_{1} d \omega_{3} d \omega_{3}}{2 \pi^{2} c^{3}} \Delta V .
\end{gathered}
$$

Here it is important to emphasize that formula (44) is correct for the observer at rest in a real space monitoring, from the referring frame, the object moving then uniformly and rectilinearly with the relativistic velocity $\mathbf{v}$.

\subsection{The Radiation Intensity Dependence on the Temperature of the Black-Body Moving Uniformly and Rectilinearly; $0 \leq \theta \leq \frac{\pi}{2}$}

Based on the aforesaid as well as on the classical methods of solving the problem (i.e., for $v<<$, see, e.g., $[9,16]$ ) we can now start its solving. For this purpose we shall write down an expression for the average total energy $\varepsilon$ of the linear oscillators as follows (Cartesian 3D-coordinate system, zero oscillations are neglected):

$$
\begin{aligned}
& \bar{\varepsilon}=\sqrt{\left(\varepsilon_{1}+\varepsilon_{2}+\varepsilon_{3}\right)}=\frac{\hbar \sum_{n_{1}=0}^{\infty} \sum_{n_{2}-0}^{\infty} \sum_{n_{3}=0}^{\infty}\left[\omega_{1} n_{1}+\omega_{2} n_{2}+\omega_{3} n_{3} e^{-\frac{\hbar \omega_{1} n_{1}}{k\left|T_{1}\right| \frac{\hbar \omega_{2} n_{2}}{k\left|T_{2}\right|}-\frac{\hbar \omega_{3} n_{3}}{k\left|T_{3}\right|}}}\right.}{\sum_{n_{1}=0}^{\infty} \sum_{n_{2}=0}^{\infty} \sum_{n_{3}=0}^{\infty} e^{-\frac{\hbar \omega_{1} n_{1}}{k\left|T_{1}\right|}-\frac{\hbar \omega_{2} n_{2}}{k\left|T_{2}\right|}-\frac{\hbar \omega_{3} n_{3}}{k\left|T_{3}\right|}}}= \\
& =\frac{\hbar \sum_{n_{1}=0}^{\infty} \omega_{1} n_{1} e^{-\frac{\hbar \omega_{1} n_{1}}{k\left|T_{1}\right|}} \sum_{n_{2}=0}^{\infty} e^{-\frac{\hbar \omega_{2} n_{2}}{k\left|T_{3}\right|}} \sum_{n_{3}=0}^{\infty} e^{-\frac{\hbar \omega_{3} n_{3}}{k\left|T_{3}\right|}}+\hbar \sum_{n_{1}=0}^{\infty} e^{-\frac{\hbar \omega_{1} n_{1}}{k\left|T_{1}\right|}} \sum_{n_{2}=0}^{\infty} \omega_{2} n_{2} e^{-\frac{\hbar \omega_{2} n_{2}}{k\left|T_{2}\right|}} \sum_{n_{3}=0}^{\infty} e^{-\frac{\hbar \omega_{3} n_{3}}{k\left|T_{3}\right|}}+\hbar \sum_{n_{1}=0}^{\infty} e^{-\frac{\hbar \omega_{1} n_{1}}{k\left|T_{1}\right|}} \sum_{n_{2}=0}^{\infty} e^{-\frac{\hbar \omega_{2} n_{2}}{k\left|T_{2}\right|}} \sum_{n_{3}=0}^{\infty} \omega_{3} n_{3} e^{-\frac{\hbar \omega_{3} n_{3}}{k\left|T_{3}\right|}}}{\hbar \omega_{1} n_{1}} \\
& \sum_{n_{1}=0}^{\infty} e^{-\frac{\hbar \omega_{1} n_{1}}{k\left|T_{1}\right|}} \sum_{n_{2}=0}^{\infty} e^{-\frac{\hbar \omega_{2} n_{2}}{k\left|T_{2}\right|}} \sum_{n_{3}=0}^{\infty} e^{-\frac{\hbar \omega_{3} n_{3}}{k\left|T_{3}\right|}} \\
& \stackrel{n_{1}, n_{2}, n_{3}=1}{=} \frac{\hbar\left(\omega_{1}+\omega_{2}+\omega_{3}\right)}{e^{\frac{\hbar \omega_{1}}{k\left|T_{1}\right|}} e^{\frac{\hbar \omega_{2}}{k\left|T_{2}\right|}} e^{\frac{\hbar \omega_{3}}{k\left|T_{3}\right|}}-e^{\frac{\hbar \omega_{1}}{k\left|T_{1}\right|}} e^{\frac{\hbar \omega_{2}}{k\left|T_{2}\right|}}-e^{\frac{\hbar \omega_{1}}{k\left|T_{1}\right|}} e^{\frac{\hbar \omega_{2}}{k\left|T_{2}\right|}}-e^{\frac{\hbar \omega_{2}}{k\left|T_{2}\right|} e^{\frac{\hbar \omega_{3}}{k\left|T_{3}\right|}}+e^{\frac{\hbar \omega_{1}}{k\left|T_{1}\right|}}+e^{\frac{\hbar \omega_{2}}{k\left|T_{2}\right|}}+e^{\frac{\hbar \omega_{3}}{k\left|T_{3}\right|}}-1}}=\frac{\hbar\left(\omega_{1}+\omega_{2}+\omega_{3}\right)}{\left(e^{\frac{\hbar \omega_{1}}{k\left|T_{1}\right|}}-1\right)\left(e^{\frac{\hbar \omega_{2}}{k\left|T_{2}\right|}}-1\right)\left(e^{\frac{\hbar \omega_{3}}{k\left|T_{3}\right|}}-1\right)}
\end{aligned}
$$

where $\omega_{1}, \omega_{2}, \omega_{3}$ are the frequencies of oscillators in the direction perpendicular and parallel to the velocity of the moving object; $n_{1}, n_{2}$ and $n_{3}$ are positive (quantum) integers for the oscillators in the first, second and third directions. In this case $n_{1}=n_{2}=n_{3}=1$, since photons are bosons, they can be in one quantum state; $T_{1}, T_{2}, T_{3}$ are the values of the temperature tensor components.

Then the average volume of the total energy $\varepsilon$ of the electromagnetic field per unit volume in the moving cavity proves to equal

$$
\varepsilon=\frac{E_{0}}{V_{0}\left(1-\beta^{2}\right)}=\frac{2 \hbar}{(2 \pi c)^{3}} \int_{0}^{\infty} \frac{\omega_{1} d \omega_{1}}{\left(e^{\frac{\hbar \omega_{1}}{k\left|T_{1}\right|}}-1\right)} \int_{0}^{\infty} \frac{d \omega_{2}}{\left(e^{\frac{\hbar \omega_{2}}{k\left|T_{2}\right|}}-1\right)} \int_{0}^{\infty} \frac{d \omega_{3}}{\left(e^{\frac{\hbar \omega_{3}}{k\left|T_{3}\right|}}-1\right)}+
$$




$$
+\frac{2 \hbar}{(2 \pi c)^{3}} \int_{0}^{\infty} \frac{\omega_{2} d \omega_{2}}{\left(e^{\frac{\hbar \omega_{2}}{k\left|T_{2}\right|}}-1\right)} \int_{0}^{\infty} \frac{d \omega_{1}}{\left(e^{\frac{\hbar \omega_{1}}{k\left|T_{1}\right|}}-1\right)} \int_{0}^{\infty} \frac{d \omega_{3}}{\left(e^{\frac{\hbar \omega_{3}}{k\left|T_{3}\right|}}-1\right)}+\frac{2 \hbar}{(2 \pi c)^{3}} \int_{0}^{\infty} \frac{\omega_{3} d \omega_{3}}{\left(e^{\frac{\hbar \omega_{3}}{k\left|T_{3}\right|}}-1\right)} \int_{0}^{\infty} \frac{d \omega_{1}}{\left(e^{\frac{\hbar \omega_{1}}{k\left|T_{1}\right|}}-1\right)} \int_{0}^{\infty} \frac{d \omega_{2}}{\left(e^{\frac{\hbar \omega_{2}}{k\left|T_{2}\right|}}-1\right)}
$$

As a result, we have obtained, in fact, nine improper integrals, three of them $\left(I^{\prime}\right)$ converge and differ only by variables. They are easily calculated using variable transformations as follows:

$$
\begin{gathered}
y_{1(2,3)}=\frac{\hbar \omega_{1(2,3)}}{\theta_{1(2,3)}}=\frac{\hbar \omega_{1(2,3)}}{k\left|T_{1(2,3)}\right|}, \\
I^{\prime}=\int_{0}^{\omega} \frac{\omega d \omega}{e^{\frac{\hbar \omega}{k|T|}}-1}=\frac{(k|T|)^{2}}{\hbar^{2}} \int_{0}^{\omega} \frac{y d y}{e^{y}-1}=\left(\frac{k|T|}{\hbar}\right)^{2} \frac{\pi^{2}}{6} .
\end{gathered}
$$

The integral of the type

$$
I^{\prime \prime}=\int_{0}^{\infty} \frac{d \omega}{\left(e^{\frac{\hbar \omega}{k|T|}}-1\right)}
$$

is integrated by quadratures but it divergences within the interval $0-\infty$, namely,

$$
I^{\prime \prime}=\frac{k|T|}{\hbar}\left[-y+\ln \left(-1+e^{y}\right)\right]_{y=0}^{y \rightarrow \infty} \rightarrow \infty, y=\frac{\hbar \omega}{k|T|},
$$

however, we can overcome the difficulties that have arisen. Indeed, we obtain the infinity for zero in the bottom limit of the integral (49). But we can take a number $\vartheta$ in the bottom limit of (49) instead zero. The number has to be very close to zero at a given temperature taking into account of the energy, by which we neglect. It must be much less than the whole energy radiated by the black body in this direction, i.e.,

$$
\vartheta<<\left[-y+\ln \left(-1+e^{y}\right)\right]_{y=\vartheta}^{y \rightarrow \infty}
$$

Here we should note that a conscious inaccuracy was made in the classical method of obtaining Stefan-Boltzmann's law. As known, according to this method, the integration takes place in the space of positive (quantum) integers. But they form a continuum for large values. If the integers are small, there is a discrete series, and we cannot formally integrate. If nevertheless we are doing that, we have:

$$
I^{\prime \prime}=\int_{\vartheta}^{\infty} \frac{d \omega}{\left(e^{\frac{\hbar \omega}{k|T|}}-1\right)}=\frac{k|T|}{\hbar} \int_{\vartheta}^{\infty} \frac{d y}{\left(e^{y}-1\right)}=\frac{k|T|}{\hbar}\left[-y+\ln \left(-1+e^{y}\right)\right]_{y=\vartheta}^{y \rightarrow \infty}=\Lambda \frac{k|T|}{\hbar} .
$$

Then we have from (46):

$$
\begin{aligned}
\varepsilon=\frac{E_{0}}{V_{0}\left(1-\beta^{2}\right)} \approx & 0,0064 a\left[\Lambda_{2} \Lambda_{3}\left|T_{1}^{2} T_{2} T_{3}\right|+\Lambda_{1} \Lambda_{3}\left|T_{2}^{2} T_{1} T_{3}\right|+\Lambda_{1} \Lambda_{2}\left|T_{3}^{2} T_{1} T_{2}\right|\right] \approx \\
& \left.\approx 0,0064 a \Lambda^{2}|| T_{1}^{2} T_{2} T_{3}|+| T_{2}^{3} T_{1} T_{3}|+| T_{3}^{2} T_{1} T_{2} \mid\right],
\end{aligned}
$$

considering that $\Lambda_{1} \approx \Lambda_{2} \approx \Lambda_{3} \approx \Lambda ; a$ is Stefan-Boltzmann's constant.

Now we are to normalize the expression (53). To do that it needs to represent (53) in the coordinate system $\mathrm{X}_{1}{ }^{\prime}, \mathrm{X}_{2}{ }^{\prime}, \mathrm{X}_{3}{ }^{\prime}$. First, the temperature vector components $T_{1}, T_{2}, T_{3}$ should be expressed in terms of the temperature vectors $T_{1}^{\prime}, T_{2}^{\prime}, T_{3}^{\prime}$. Having done that, we shall have: 


$$
\left.\begin{array}{l}
T_{1}=T_{1}^{\prime} \cos \theta+T_{2}^{\prime} \sin \theta \\
T_{2}=-T_{1}^{\prime} \sin \theta+T_{2}^{\prime} \cos \theta \\
T_{3}=T_{3}^{\prime}
\end{array}\right\},
$$

and

$$
\left.\begin{array}{l}
\left|T_{1}\right|=\left|T_{1}^{\prime} \cos \theta+T_{2}^{\prime} \sin \theta\right| \\
\left|T_{2}\right|=\left|-T_{1}^{\prime} \sin \theta+T_{2}^{\prime} \cos \theta\right| \\
\left|T_{3}\right|=\left|T_{3}^{\prime}\right|
\end{array}\right\} .
$$

Second, the quantities $\left|T_{1}^{\prime}\right|,\left|T_{2}^{\prime},\right| T_{3}^{\prime} \mid$ should be expressed in term of $\left|T_{01}^{\prime},\right|,\left|T_{02}^{\prime},\right|,\left|T_{03}^{\prime}\right|$, which are vector temperature components when $\mathbf{v}=0$. We must also take into account of dependences (41) for the angle $\theta$ [9].

Substituting (55) into (53), we obtain taking into account of (25):

$$
\begin{aligned}
\left.\varepsilon=\frac{E_{0}}{V_{0}\left(1-\beta^{2}\right)} \approx 0,0064 a \Lambda^{2}|| T_{1}^{2} T_{2} T_{3}|+| T_{2}^{3} T_{1} T_{3}|+| T_{3}^{2} T_{1} T_{2} \mid\right] \approx 0,0064 a \Lambda^{2} \mathrm{x} \\
\times\left[\left|T_{03}\right|\left|\frac{T_{01}^{\prime}}{\sqrt{1-\beta^{2}}} \cos \theta+T_{02}^{\prime} \sin \theta\right| \mid-\frac{T_{01}^{\prime}}{\sqrt{1-\beta^{2}}} \sin \theta+T_{02}^{\prime} \cos \theta+\right. \\
+\left|T_{03}\right|\left|-\frac{T_{01}^{\prime}}{\sqrt{1-\beta^{2}}} \sin \theta+T_{02}^{\prime} \cos \theta\right|\left|\frac{T_{01}^{\prime}}{\sqrt{1-\beta^{2}}} \cos \theta+T_{02}^{\prime} \sin \theta\right|+ \\
\left.+\left|T_{03}\right|^{2}\left|\frac{T_{01}^{\prime}}{\sqrt{1-\beta^{2}}} \cos \theta+T_{02}^{\prime} \sin \theta\right|-\frac{T_{01}^{\prime}}{\sqrt{1-\beta^{2}}} \sin \theta+T_{02}^{\prime} \cos \theta \mid\right]
\end{aligned}
$$

At $\mathrm{v}=0$, a numerical coefficient in (53) must be equal to $a$, since (53) has to be transformed in Stefan-Boltzmann's law under the normal conditions (3).

Thus an additional multiplier in the right side of (53) must be equal to 1. Of course, the expression (56) is cumbersome, however we can normalize it at any angle $\theta$ using computer. Without the computer we shall do it for the angles 0 and $\pi / 2$ (see Fig.2).

If $\theta=0$ and, consequently, $\sin \theta=\sin \theta_{0}$ (see (41)), we have from (56)

$$
\varepsilon=\frac{E_{0}}{V_{0}\left(1-\beta^{2}\right)} \approx 0,0064 \Lambda^{2} a\left[\frac{T_{01}^{\prime}}{\sqrt{1-\beta^{2}}} T_{02}^{\prime} T_{03}^{2}+\frac{T_{01}^{\prime 2}}{1-\beta^{2}} T_{02}^{\prime} T_{03}+\frac{T_{01}^{\prime}}{\sqrt{1-\beta^{2}}} T_{02}^{\prime} T_{03}\right],
$$

and

$$
\varepsilon=\frac{E_{0}}{V_{0}\left(1-\beta^{2}\right)} \approx 0,0064 \Lambda^{2} a\left[\frac{2 T_{01}^{\prime}}{\sqrt{1-\beta^{2}}} T_{02}^{\prime 2} T_{03}+\frac{T_{01}^{\prime 2}}{1-\beta^{2}} T_{02}^{\prime} T_{03}\right],
$$

since if $\theta=0$, so $T_{02}^{\prime}=T_{03}^{\prime}=T_{03}$. In (57), (58), and below in (65) all the temperature $T$ components are the absolute values of this quantity, e.g., $T_{01}^{\prime}=\left|T_{01}^{\prime}\right|$.

If, in turn, $\mathbf{v}=0$, we shall have from (58):

$$
\varepsilon \approx 0,0192 \Lambda^{2} a T_{0}^{\prime 4}
$$


then $0,0192 \Lambda^{2}$ should be equal to 1 , consequently, $\Lambda^{2}=52.1$, and $\Lambda=7.22$.

Now we must find the above quantity $\vartheta$ (see (52). First, we should solve a transcendent equation

$$
\Lambda=7.22=\vartheta-\ln \left(-1+e^{\vartheta}\right),
$$

its solution (see Appendix below) is:

$$
\vartheta=-\ln \left(1-e^{-\Lambda}\right\}
$$

Then $\vartheta=0.00073$. Evidently, if the quantity $\vartheta$ is so small, so we neglect a very small energy of the object under study calculating the improper integral like (49). Indeed, let $T_{1}^{\prime}$ be $5000 \mathrm{~K}$, then $\frac{\hbar \omega_{0}}{k T_{0}^{\prime}}=0,00073$, and $\omega_{0}=7.60 \times 10^{10} \mathrm{~Hz}$, i.e., first of all. we have neglected by the energy of the low power waves. Then finally (58) will take the form

$$
\varepsilon=\frac{E_{0}}{V_{0}\left(1-\beta^{2}\right)} \approx a\left[0.67 \frac{T_{01}^{\prime}}{\sqrt{1-\beta^{2}}} T_{02}^{\prime 3}+0.33 \frac{T_{01}^{\prime 2}}{1-\beta^{2}} T_{02}^{\prime 2}\right] .
$$

As to the second case, i.e., the case, when the angle $\theta=\frac{\pi}{2}$, so the formulae (57), (58) and (62) will undergo a change: they read:

$$
\begin{gathered}
\varepsilon=\frac{E_{0}}{V_{0}\left(1-\beta^{2}\right)} \approx 0,0064 \Lambda^{2} a\left[\frac{T_{02}^{\prime}}{\sqrt{1-\beta^{2}}} T_{01}^{\prime} T_{03}^{2}+\frac{T_{02}^{\prime 2}}{1-\beta^{2}} T_{01}^{\prime} T_{03}+\frac{T_{02}^{\prime}}{\sqrt{1-\beta^{2}}} T_{01}^{\prime} T_{03}\right] \\
\varepsilon=\frac{E_{0}}{V_{0}\left(1-\beta^{2}\right)} \approx 0,0064 \Lambda^{2} a\left[\frac{2 T_{02}^{\prime}}{\sqrt{1-\beta^{2}}} T_{01}^{\prime 2} T_{03}+\frac{T_{02}^{\prime 2}}{1-\beta^{2}} T_{01}^{\prime} T_{03}\right] \\
\varepsilon=\frac{E_{0}}{V_{0}\left(1-\beta^{2}\right)} \approx a\left[0.67 \frac{T_{02}^{\prime}}{\sqrt{1-\beta^{2}}} T_{01}^{\prime 3}+0.33 \frac{T_{02}^{\prime 2}}{1-\beta^{2}} T_{01}^{\prime 2}\right] .
\end{gathered}
$$

This a conclusion follows directly from (57).

As seen from (62) and (65), almost all radiation of the object will be directed parallel to the vector of its motion as $v \rightarrow c$, since, e.g., $\frac{T_{02}^{\prime}}{\sqrt{1-\beta^{2}}}$ will much exceed $T_{01}^{\prime}$ and $T_{03}$. This conclusion is in a full agreement with relativistic electrodynamics [9].

The numerical coefficients in (65), i.e., 0.67 and 0.33 , differ on the numerical ones in (40), i.e., 0,79 and 0.21 . It is naturally, since there are six improper singular integrals in (46) but there is only one such integral in (29).

\section{Discussion}

It should be noted at once that the dependence (56) does not lead to the point of absurdity and contradictions. The dependence (56) provides rather a probable answer to the question concerning the temperature transformation under relativistic conditions. It is evident that the dependence (1) is incorrect and would be rejected many years ago and without all mathematical involvements if it were not a giant authority of Planck and Einstein. Really, how is the dependence (1) be followed if such cosmic objects as quasars do exist, whose velocity $v$ of motion can be equal $0,93 c$ with the luminosity reaching enormous values?

In the most general case the temperature is a complex mathematical object. It comprises an invariant part independent of the motion velocity and a part dependent on the velocity and oriented in space. Under normal conditions the temperature becomes a scalar, the same does for the heat. The entropy problem has not been studied completely. It is not improbable that the entropy can be a tensor object in which indices are contracted which results in a scalar independent of the system motion velocity. Evidently, it is for experiment to solve this problem. However no experiment has been performed since the birth of relativistic thermodynamics in 1907.

Of utmost interest is to consider if the dependence (20) remains valid for the case of an ultrarelativistic high-temperature spherical plasma (fireball) [11]. According to the author of [11], the spectrum of its equilibrium radiation $d \varepsilon_{\gamma}\left(\omega_{*}\right)(\mathrm{J} / \mathrm{cc})$ due to the annihilation of 
electrons and positrons is described by the dependence

$$
d \varepsilon_{\gamma}\left(\omega_{*}\right)=\frac{T^{4}}{\pi^{2} \hbar^{3} c^{3}} \frac{\omega_{*}^{2} \sqrt{\omega_{*}^{2}-\Delta^{2} f}}{e^{\omega_{*}}-1} d \omega_{*},
$$

in the fireball, where $\omega_{*}=\hbar \omega / T$ is the dimensionless frequency; $T \gg m c^{2}$ is the energy, i.e., apparently, $\theta=k T$ ( $k$ is the Boltzmann constant), $T$ is now the absolute temperature; $\theta$ not to be confused with the angle similarly designated (see above); $\Delta=\hbar \omega_{p, r e l} / T ; \omega_{p, r e l}$ is the relativistic frequency of the plasma oscillations; $f$ is a dimensionless constant.

Formula (20) is valid for the case (66) with the vector part of the temperature dependent on the total velocity of electrons and positrons in the fireball but not on the velocity of its centre of mass. If their velocities are very high, then we have the well-known case described, e.g., in [9]. This is the case of a system of particles being widely apart and moving with very high velocities. It should be noted that these two cases are not fully identical, since the microparticles in [9] are not identical before and after the collision. In article [11], an electron-positron collision results in their annihilation. However these cases are very similar, thus the system energy $\varepsilon$ may be given as

$$
\varepsilon \sim \sum \frac{m_{i} c^{2}}{\sqrt{1-\frac{v_{i}^{2}}{c^{2}}}},
$$

where $m_{i}$ is the microperticle mass, $v_{i}$ is its velocity. Then the vector part of the temperature in the ultrarelativistic case will transform in inverse proportion of the roots $\sqrt{1-v_{i}^{2} / c^{2}}$. Here we immediately arrive at the conclusion that the dependence (66) is very doubtful, since the right side does not transform identically to its left side under the relativistic conditions. It should be also noted that the object studied in [11] is, in fact, a stable fireball. Evidently, when the density of electrons and positrons exceeds a certain limit, the stability will be broken, and an explosion will occur.

\section{Conclusions}

Laws were obtained for the black-body radiation in the whole interval of its (black-body) movement speed, i.e., from zero up to the speed of light in vacuum.

The first law is a special case when an angle $\theta$ between the movement velocity of the object under study and the observer is equal to zero. The second law is a generalized case when the angle $\theta$ can vary within the interval from zero up to $\pi / 2$ inclusively.

\section{Appendix. Obtaining the Solution of (60)}

We have from (60)

$$
\begin{gathered}
\ln e^{\vartheta}-\ln \left(-1+e^{\vartheta}\right)=\ln e^{\Lambda}, \\
\ln \left[\frac{e^{\vartheta}}{-1+e^{\vartheta}}\right]=\ln e^{\Lambda}, \\
{\left[\frac{e^{\vartheta}}{-1+e^{\vartheta}}\right]=e^{\Lambda},} \\
e^{-\Lambda} e^{\vartheta}=-1+e^{\vartheta}, \\
\left(e^{-\Lambda} e^{\vartheta}-e^{\vartheta}\right)=-1, \\
e^{\vartheta}\left(e^{-\Lambda}-1\right)=-1,
\end{gathered}
$$

and finally

$$
\vartheta=\ln \left(-\frac{1}{e^{-\Lambda}-1}\right)=\ln \left(\frac{1}{1-e^{-\Lambda}}\right)=-\ln \left(1-e^{-\Lambda}\right\} \text {, i.e (61). }
$$

\section{REFERENCES}

[1] K. von Mosengeil. Theorie der stationaren Strahlung in einem gleichformig bewegten Hohlraum, Annalen der Physik, Vol.22, №5, 867 - 904, 1907

[2] M. Planck. Zur Dinamik bewegter Systemen, Annalen der Physik, Vol. 26, №6, 1- 34, 1908.

[3] X. Ott. Lorenz-Transformation der Waerme and der Temperatur, Zeitschrift f. Phys.,Vol.175, №1, 70-104, 1963.

[4] H. Callen, G. Horwitz. Relativistik Thermodynamics, Am. J. Phys., Vol.39, №8, 938 - 947, 1971.

[5] G.Cavalleri, G. Salgarelli. Revision of the relativistic dynamics with variable rest mass and application to relativistic thermodynamics, It nuovo cimento. Organo della societa italiano di fisica, ser.10, Vol. A62, №№ $2-4,722$ 754, 1969.

[6] P.T. Landsberg, G.E.A. Matsas. Laying the ghost of the relativistic Transformation, Physics Letters A, Vol, 223, 401-403, 1996.

[7] P.T. Landsberg, G.E,A. Matsas. The impossible of an universal relativistic temperature transformating, Physica A, Vol, 340, №№1 - 3, 92 - 94, 2004.

[8] A. Einstein. Ueber das Relativitaetsprinzip und die aus demselbe gezogenen Folgerungen, Jahrbuch deutsche Radioaktivitaet und Elektronik, №4: 411 - 462, 1907.

[9] V.G. Levich. The Course of Theoretical Physics, V.1., Fizmatgiz, Moscow, 1963.

[10] V.H. Hamity, Relativistic Thermodynamics, Phys. Rev., Vol. 187, №5, 1745 - 1752, 1969.

[11] M.V. Medvedev. Thermodynamics of photon in relativistic e+e- $\gamma$ plasma, Phys. Review E., Vol. 59, №5, 4766 - 4768, 1999. 
[12] R. Hakim, A. Mangeney. Remarks on Relativistic Thermodynamics, Lettere al Nuovo Cimento. Revista internazionale Society della italiana Di Fisica, seria 1,Vol.1, №9, $429-435,1969$.

[13] E.V. Veitsman. Some Problems in Relativistic Thermodynamic, J. Experimental and Theoretical Phys., 105, №5, 1057 - 1067, 2007.

[14] E.V.Veitsman. On Relativistik Surface Tension, J. Colloid
Interface Sci., 265, №1, 174 178, 2003.

[15] E.V. Veitsman. Specific thermodynamical potentials on surfaces under relativistic conditions, J Colloid Interface Sci.,Vol. 337, №2, 355-357,2009.

[16] Y.P. Terletsky. Statistical Physics, Visshaya Shkola, Moscow,1966.

[17] A.P. Prudnikov, U.A. Brychkov, O.I. Marichev. Integrals and Series, V.1 (Page 177), Fizmatgiz, Moscow, 2003. 